\title{
Estructura de tallas, selectividad y composición específica de las capturas en trampas para peces marinos en el Golfo de California
}

\author{
Manuel O. Nevárez-Martínez, Alejandro Balmori-Ramírez, Everardo Miranda-Mier, J. Pablo \\ Santos-Molina, Francisco J. Méndez-Tenorio \& Celio Cervantes-Valle \\ Instituto Nacional de Pesca. Unidad Guaymas. Calle 20 Sur No. 605. Col. Centro, C. P. 85 400, Guaymas, Sonora, \\ México; manuel.nevarez@prodigy.net.mx
}

Recibido 14-IX-2007. Corregido 07-V-2008. Aceptado 26-V-2008.

\begin{abstract}
Size structure, selectivity and specific composition of the catch in traps for marine fish in the Gulf of California. We analyzed the performance of three traps for marine fish between October 2005 and August 2006 in the Gulf of California, Mexico. The performance was measured as difference in selectivity, fish diversity, size structure and yield. The samples were collected with quadrangular traps $90 \mathrm{~cm}$ wide, $120 \mathrm{~cm}$ long and $50 \mathrm{~cm}$ high. Trap type 1 had a $5 \times 5 \mathrm{~cm}$ mesh (type $2: 5 \times 5 \mathrm{~cm}$ including a rear panel of $5 \times 10 \mathrm{~cm}$; trap 3:5 $\mathrm{x} 10 \mathrm{~cm}$ ). Most abundant in our traps were: Goldspotted sand bass (Paralabrax auroguttatus), Ocean whitefish (Caulolatilus princeps), Spotted sand bass (P. maculatofaciatus) and Bighead tilefish (C. affinis); there was no bycatch. The number of fish per trap per haul decreased when mesh size was increased. We also observed a direct relationship between mesh size and average fish length. By comparing our traps with the authorized fishing gear (hooks-and-line) we found that the size structure is larger in traps. Traps with larger mesh size were more selective. Consequently, we recommend adding traps to hooks-and-line as authorized fishing gear in the small scale fisheries of the Sonora coast, Mexico. Rev. Biol. Trop. 56 (3): 1403-1417. Epub 2008 September 30.
\end{abstract}

Key words: fish traps, hooks-and-line, gear selectivity, Gulf of California, Mexico.

Aun cuando en México las trampas se utilizan para capturar peces demersales (Anónimo 2000), con excepción de las pesquerías de langosta y jaiba, no existe una pesquería establecida que utilice las trampas como el arte de pesca principal. Sin embargo, lugares como New South Wales, Australia, tienen pesquerías demersales multiespecíficas con trampas para peces, con desembarques de 700 toneladas, que representan aproximadamente US\$3.5 millones y dan empleo a 400 pescadores (Stewart y Ferrell 2003). En aguas del conteniente americano se capturan peces con trampas, e.g., -USA, Venezuela, Bermuda, Trinidad y Tobago (Smith 1971, Bullock y Murphy 1994, Manickchand-Heileman y Phillip 2000) y en el Caribe (Wolf y Chislett 1974, Munro 1983, Nicholson y Hartsuijker 1983, Luckhurst y
Ward 1987, Mahon y Hunte 2001). Los peces asociados a este tipo de pesquería pueden ser de alto valor comercial, como los meros, pargos y cabrillas (Manickchand-Heileman y Phillip 2000, Mahon y Hunte 2001, Stewart y Ferrell 2003).

En México esas especies son un componente muy importante de la pesca de escama marina, la cual se captura en la plataforma continental hasta profundidades de $200 \mathrm{~m}$, mediante diversas artes de pesca (red de arrastre, de enmalle, palangre o líneas de mano), pero raramente con trampas (Anónimo 2000). Este grupo de peces presentan una alta variabilidad interanual en sus capturas, que no exceden las dos mil toneladas anuales (Anónimo 2000) en Sonora.

Considerando la magnitud, el precio en el mercado nacional y la gran proporción de pesca 
incidental en las capturas (Anónimo 2000), es primordial realizar un estudio orientado al diseño y experimentación de un arte de pesca que permita, mantener vivos a los organismos, seleccionar los tamaños de los peces que se desembarquen, y liberar vivos a aquellos especímenes que no cumplan con las regulaciones correspondientes o a la fauna asociada, y que abra la oportunidad de que se ofrezcan vivos en el mercado internacional, con el fin de aumentar su valor comercial y socio-económico en la región. En este trabajo se presentan los aspectos asociados a la eficiencia de captura, estructura de tallas, selectividad y composición de especies en trampas de diferentes tamaños de malla para la pesca de peces marinos en el Golfo de California.

\section{MATERIALES Y MÉTODOS}

El trabajo de campo se realizo en tres localidades de la zona costera del centro-sur del estado de Sonora, en el Golfo de California, México: área de Kino-Isla Tiburón (28 $8^{\circ} 25^{\prime}$ a $28^{\circ} 37^{\prime} \mathrm{N} ; 111^{\circ} 48^{\prime}$ a $112^{\circ} 38^{\prime} \mathrm{W}$ ), área de Guaymas $\left(27^{\circ} 37^{\prime}\right.$ a $28^{\circ} 01^{\prime} \mathrm{N} ; 110^{\circ} 27^{\prime}$ a $111^{\circ}$ $1^{\prime}$ W) y área de Yavaros $\left(26^{\circ} 38^{\prime}\right.$ a $27^{\circ} 00^{\prime} \mathrm{N}$; $109^{\circ} 31^{\prime}$ a $110^{\circ} 00^{\prime} \mathrm{W}$ ).

La recolecta de muestras se efectuó con trampas de $90 \mathrm{~cm}$ de ancho, $120 \mathrm{~cm}$ de longitud y $50 \mathrm{~cm}$ de alto, con malla galvanizada cuadrangular en tres tamaños: una trampa completamente con abertura de $5 \times 5 \mathrm{~cm}$ (trampa 1); otra con malla de $5 \times 5 \mathrm{~cm}$, pero con el panel trasero de $5 \times 10 \mathrm{~cm}$ (trampa 2) y la tercera con malla de $5 \times 10 \mathrm{~cm}$ (trampa 3) (Nevárez-Martínez et al. 2005). Cada tipo cuenta con bocas a los lados, una cámara hecha de malla de cuadro pequeño como encarnador, y una pared de malla de 5 x $5 \mathrm{~cm}$ con una boca en medio para que los peces pasen de la cámara de entrada a la cámara de retención, de modo que impide que los peces regresen por donde entraron (Fig. 1). La trampa tiene un cabo, cuyo extremo está amarrado a una boya, para engancharla y extraerla del agua (NevárezMartínez et al. 2005). Por otro lado, en el área de Kino, se adicionaron dos tipos de trampas: trampa 4 con diseño similar a la trampa 1 , pero con menos anchura $(70 \mathrm{~cm}$ en vez de $90 \mathrm{~cm}$ ) y de otro material (varilla cubierta de plástico negro). La trampa 5 también es similar a la trampa 1 (malla de $5 \times 5 \mathrm{~cm}$ y dimensiones muy similares), pero está construida de paño y es abatible.

Los experimentos se llevaron a cabo entre octubre del 2005 y agosto del 2006. Las trampas fueron encarnadas con sardina monterrey (Nevárez-Martínez et al. 2005) y se colocaron en el fondo, en profundidades de 40 a $150 \mathrm{~m}$. Una vez colocadas, se revisaron cada hora por periodos de pesca de hasta seis horas. La captura obtenida en cada trampa se contó y pesó en su totalidad.

Posteriormente, se determinó la composición por especie con las claves propuestas por Fischer et al. (1995), INP (1976), Miller y Lea (1972) y Thomson et al. (1979) y se registró la captura de cada una de ellas. El muestreo biométrico de las especies más abundantes (y valiosas), incluyó la longitud patrón y total $(\mathrm{mm})$. Se midieron todos los ejemplares capturados en las trampas para determinar la estructura de tallas en la captura y realizar el análisis de selectividad.

Para los análisis de selectividad se utilizaron tres diferentes tamaños de malla, dado que la selectividad de un equipo de pesca se puede describir mediante la comparación de las curvas de selección de dos o más tipos de mallas diferentes (Wileman et al. 1996, Millar y Fryer 1999, Stuart y Ferrell 2003). La trampa 1, por tener la malla más pequeña, se utilizó como el testigo o control (Jeong et al. 2000, Stuart y Ferrell 2003, Akiyama et al. 2004).

Aunque existen varios métodos para estimar y analizar la selectividad de las artes de pesca (Pope et al. 1975, Hovgard 1996, Wileman et al. 1996, Helser et al. 1998, Millar y Fryer 1999, Stuart y Ferrell 2003), en este estudio se utilizó el método SELECT (Share Each LEngth's Catch Total) para el análisis de datos de los experimentos de selectividad (Millar 1992, 1995, Millar y Walsh 1992, Xu y Millar 1993, Wileman et al. 1996, Millar y Fryer 1999, Jeong et al. 2000, Stuart y Ferrell 2003). 


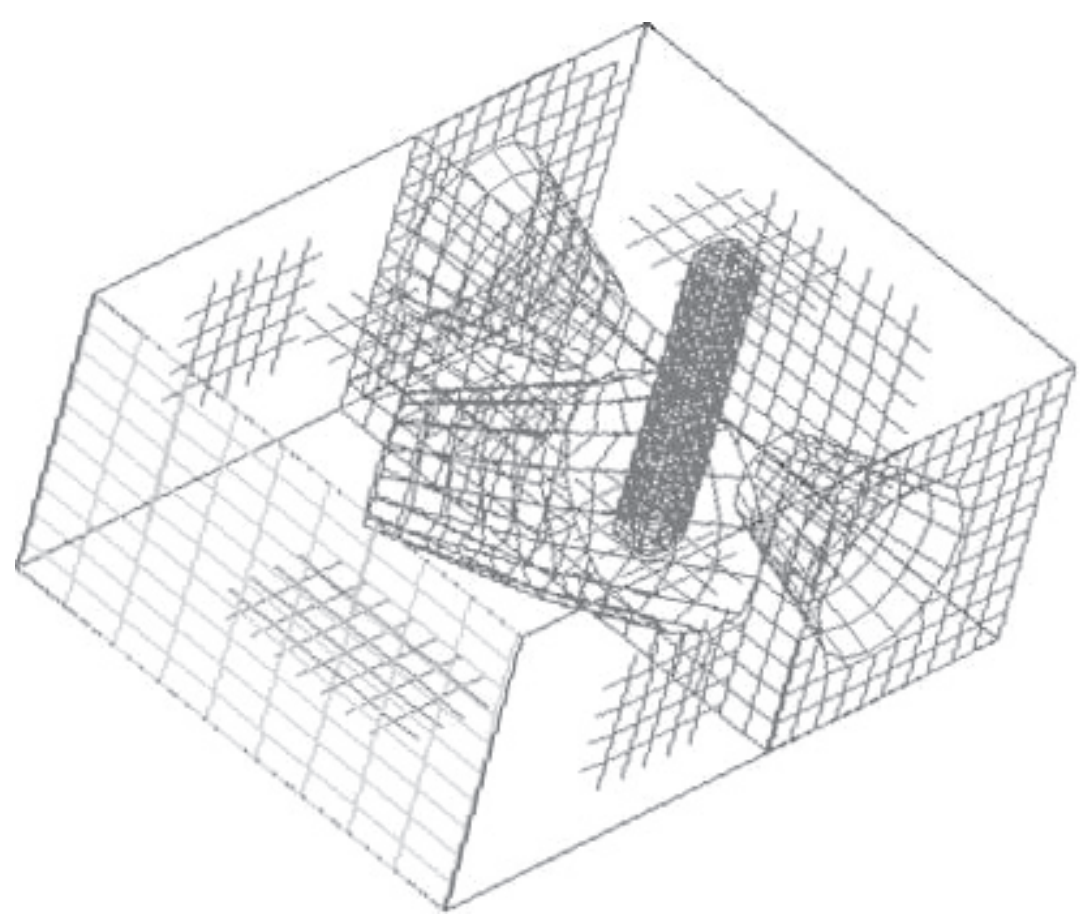

Fig. 1. Trampa de $5 \times 5 \mathrm{~cm}$, con panel trasero de malla de $5 \times 10 \mathrm{~cm}$, para la pesca de peces marinos.

Fig. 1. Trap of $5 \times 5 \mathrm{~cm}$, with back panel with mesh of $5 \times 10 \mathrm{~cm}$, for fishing marine fish.

En el modelo SELECT, la proporción, $R_{l}$, de la captura de peces de longitud $l$ retenidos por el equipo experimental con respecto a la captura total es expresada por la siguiente ecuación:

$$
R_{l}=n_{i l} /\left(n_{i l}+n_{c l}\right)
$$

donde $n_{i l}$ y $n_{c l}$ son el número de peces de longitud $l$ retenidos en el equipo experimental y el control, respectivamente. La proporción capturada en el $i$-ésimo equipo experimental con respecto a la captura total de peces de longitud $l$, está dada por:

$$
R_{i}(l)=\left(p_{i}{ }^{*} r(l)\right) /\left(p_{i}{ }^{*} r(l)+1-p_{i}\right)
$$

donde $r(l)$ es la probabilidad de que un pez de longitud $l$ sea retenido y $p_{i}$ es un parámetro independiente de la longitud $l$ del pez del $i$-ésimo equipo experimental, que puede ser definido como el poder relativo de pesca y puede ser dividido (denotado en la literatura como parámetro "split") en: a) esfuerzo relativo de pesca y b) eficiencia relativa de pesca de los tipos de trampas, dado que todos los peces capturados fueron medidos (Xu y Millar 1993, Millar y Fryer 1999, Stuart y Ferrell 2003).

La curva de selectividad puede ser definida con la función logística simétrica (Xu y Millar 1993, Millar y Fryer 1999, Stuart y Ferrell 2003):

$$
r(l)=\left[\exp ^{\left(a-b^{*} \mathrm{~L}\right)} /\left(1+\exp ^{\left(a-b^{*} \mathrm{~L}\right)}\right)\right]
$$

donde $a$ y $b$ son los parámetros de la función logística, que caracterizan a la curva de selectividad (Millar y Fryer 1999, Jeong et al. 2000, Akiyama et al. 2004). Esto permitió estimar la ojiva de selección $L_{50 \%}$, la longitud a la cual el $50 \%$ de los peces son retenidos en las trampas, y el ámbito de selección $(R S)$, entendido como 
la diferencia entre $L_{75 \%}$ y $L_{25 \%}$, se estimaron de la siguiente manera:

$$
\begin{gathered}
L_{50 \%}=-a / b \quad(4), \\
R S=L_{75 \%}-L_{25 \%}=(2 * \operatorname{Ln}(3)) / b
\end{gathered}
$$

Los parámetros de la curva de selectividad y el parámetro $p_{i}$ fueron estimados, bajo el supuesto de que las proporciones observadas están distribuidas binomialmente (Millar y Walsh 1992, Wileman et al. 1996, Jeong et al. 2000), con métodos de máxima verosimilitud. El logaritmo de la verosimilitud a ser maximizado es:

$$
\log L_{i}=\sum_{l}\left[n_{i l} \ln R_{i}(l)+n_{c l} \ln \left(1-R_{i}(l)\right]\right.
$$

Se usó la función SOLVER en MS-Excel para la maximización de la función de logaritmo de verosimilitud (Tokai 1997).

\section{RESULTADOS}

Las especies que presentaron mayor incidencia de captura en cuanto a proporción del volumen total fueron: extranjero (Paralabrax auroguttatus Walford, 1936), pierna (Caulolatilus princeps Jenyns, 1840), cabrilla arenera (P. maculatofaciatus Steindachner, 1868) y conejo (C. affinis Gill, 1865). También se capturaron otras especies de importancia comercial, pero en cantidades muy pequeñas, las cuales pertenecen a los géneros Lutjanus Bloch, 1790; Balistes Linnaeus, 1758; Epinephelus Bloch, 1790; Nebris Cuvier, 1830; Scorpaena Linnaeus, 1758; Scarus Forsskal, 1775; Sphoeroides Anonymous [Lacepede], 1798; Calamus Swainson, 1839 y Octopus Cuvier, 1797. Cabe mencionar tres especies cuya captura podría considerarse como incidental, tanto por su baja presencia como por no tener valor comercial: tres especímenes de tiburón gata (Heterodontus mexicanus Taylor y Castro-Aguirre, 1972), una anguila o morena
(Muraenidae) y un rojillo (Scorpaena $s p$.). Según estos resultados, prácticamente todas las capturas tuvieron valor comercial. La anguila y uno de los tiburones gata se pudieron liberar vivos.

El Cuadro 1 muestra el número promedio de peces capturados por trampa por día (y piola con anzuelo) y localidad, así como la talla promedio de los peces capturados. La trampa 2 no registró capturas, en ninguna de las localidades. La trampa 1 presentó una captura 1.6 y 10 veces mayor que la trampa 3 . Por otra parte, en Kino, la trampa 1 capturó $25 \%$ menos que la trampa 4 , pero capturo 4.7 veces más que la trampa 5. La longitud promedio general fue muy variable, reflejando las variaciones de las tallas medias para las principales especies, dependiendo de la localidad: en Kino la trampa 3 (malla grande) atrapó peces más grandes que los otros tipos de trampas, por lo que la longitud media fue mayor en la trampa de malla grande. Sin embargo en Guaymas aunque la malla grande capturó organismos más grandes en algunas especies (e.g., extranjero), no fue así en la pierna. Finalmente, en Yavaros la talla media fue más pequeña en la trampa 3 (e.g., conejo) (Cuadro 1).

Las estructuras de tallas generales en las capturas, por localidad y por tipo de trampa y por especie se muestran en las figuras 2 a 6 . En Guaymas, la trampa 1 presentó un ámbito mayor que el observado en la trampa 3, situación que también se observó en Yavaros (Fig. 2 y 3). En Kino, la estructura de tallas fue similar entre la trampa 1 y 4, presentándose proporcionalmente organismos más grandes en las trampas 3 y 5 (Fig. 4). En el caso del extranjero, en Guaymas, la trampa 1 capturó peces en un ámbito de tallas más amplio que la trampa 3, la cual atrapó proporcionalmente organismos más grandes (Fig. 5). Por otro lado, la estructura de tallas de la pierna en las trampas 1 y 3 fue muy similar (Fig. 5).

En la zona de Kino, la trampa 1 y 4 capturaron extranjeros con una distribución de frecuencias de tallas similares, con la trampa 3 atrapando los organismos más grandes (Fig. 6A). La estructura de tallas de la pierna en 
CUADRO 1

Número total promedio de peces capturados por trampa (y piola con anzuelos) por día de pesca y longitud media general ( \pm desviación estándar, sd) y por principales especies capturadas en tres localidades de la costa de Sonora, México.

2005-2006

TABLE 1

Total number average of fish captured by trap (and hooks-and-line) per day of fishing and general average length ( \pm standard deviation, sd) and by main species captured in three localities of the Sonora coast, Mexico. 2005-2006

$\begin{array}{ccccccc} & \text { Total } & \text { Extranjero } & \text { Pierna } & \text { Conejo } & \text { Cabrilla } \\ \text { Localidad y tipo trampa } & \begin{array}{c}\text { Peces por } \\ \text { trampa }\end{array} & \begin{array}{c}\text { Media } \pm \mathrm{sd} \\ (\mathrm{mm})\end{array} & \begin{array}{c}\text { Media } \pm \mathrm{sd} \\ (\mathrm{mm})\end{array} & \begin{array}{c}\text { Media } \pm \mathrm{sd} \\ (\mathrm{mm})\end{array} & \begin{array}{c}\text { Media } \pm \mathrm{sd} \\ (\mathrm{mm})\end{array} & \begin{array}{c}\text { Media } \pm \mathrm{sd} \\ (\mathrm{mm})\end{array}\end{array}$

Kino

\begin{tabular}{|c|c|c|c|c|c|c|c|c|c|c|c|}
\hline Trampa $5 \times 5$ & 61 & 297 & \pm 42 & 287 & \pm 41 & 308 & \pm 36 & 278 & \pm 35 & 248 & \pm 41 \\
\hline Trampa $5 \times 10$ & 0 & 0 & 0 & 0 & 0 & 0 & 0 & 0 & 0 & 0 & 0 \\
\hline Trampa $5 \times 5-5 \times 10$ & 6 & 360 & \pm 48 & 356 & \pm 32 & 376 & \pm 39 & 363 & 37 & & \\
\hline Trampa $5 \times 5$ plegable & 13 & 316 & \pm 32 & 312 & \pm 31 & 319 & \pm 38 & & & & \\
\hline Trampa $5 \times 5$ plastificada & 80 & 286 & \pm 44 & 287 & \pm 49 & 299 & \pm 35 & 279 & \pm 33 & 239 & \pm 24 \\
\hline Piola & 42 & 273 & \pm 40 & 273 & \pm 49 & 282 & \pm 36 & & & & \\
\hline \multicolumn{11}{|l|}{ Guaymas } & \\
\hline Trampa $5 \times 5$ & 13 & 295 & \pm 40 & 282 & \pm 43 & 305 & \pm 35 & 291 & \pm 42 & & \\
\hline Trampa $5 \times 10$ & 0 & 0 & 0 & 0 & 0 & 0 & 0 & 0 & 0 & & \\
\hline Trampa $5 \times 5-5 \times 10$ & 8 & 299 & \pm 39 & 308 & \pm 53 & 299 & \pm 30 & & & & \\
\hline Piola & 12 & 289 & \pm 51 & 277 & \pm 49 & 311 & \pm 40 & 241 & \pm 41 & & \\
\hline \multicolumn{11}{|l|}{ Yavaros } & \\
\hline Trampa $5 \times 5$ & 7 & 264 & \pm 33 & & & & & 273 & \pm 26 & & \\
\hline Trampa $5 \times 10$ & 0 & 0 & 0 & & & & & 0 & 0 & & \\
\hline Trampa $5 \times 5-5 \times 10$ & 2 & 251 & \pm 26 & & & & & 260 & \pm 17 & & \\
\hline Piola & 5 & 390 & \pm 99 & & & & & 273 & \pm 28 & & \\
\hline
\end{tabular}

las trampas mostró el mismo comportamiento que en el extranjero, es decir los organismos más grandes fueron capturados por la trampa con malla más grande (Fig. 6B). En el caso del conejo, la trampa 1 y 4 atraparon peces en un ámbito de tallas parecido, mientras que la trampa 3 capturó a los organismos más grandes (Fig. 6C). En cuanto a la cabrilla arenera, la estructura de tallas obtenida en la trampas 1 y 4 fue semejante, aunque la trampa 1 obtuvo una proporción mayor de organismos grandes (Fig. 6D).

Con base en la información mostrada en las figuras 5 y 6 , se estimaron las curvas de selección utilizando la información de las trampas 1, 3, 4 y 5. En todos los casos la selectividad fue estimada por comparar los peces retenidos en las trampas 3, 4 y 5 con respecto a los retenidos en la trampa 1. En el Cuadro 2 se muestran los estimados de los parámetros de la curva de selectividad, la longitud del 50\% de retención $\left(L_{50 \%}\right)$ y el ámbito de selección $(R S)$. La $L_{50 \%}$ estimada se incrementó con el tamaño de malla, para el extranjero, pierna y conejo, en la zona de Kino, mientras que la $L_{50 \%}$ estimada para la trampa $3(5 \times 5-5 \times 10)$, tanto para el extranjero como para la pierna en Guaymas, fue menor que la estimada para estas especies 

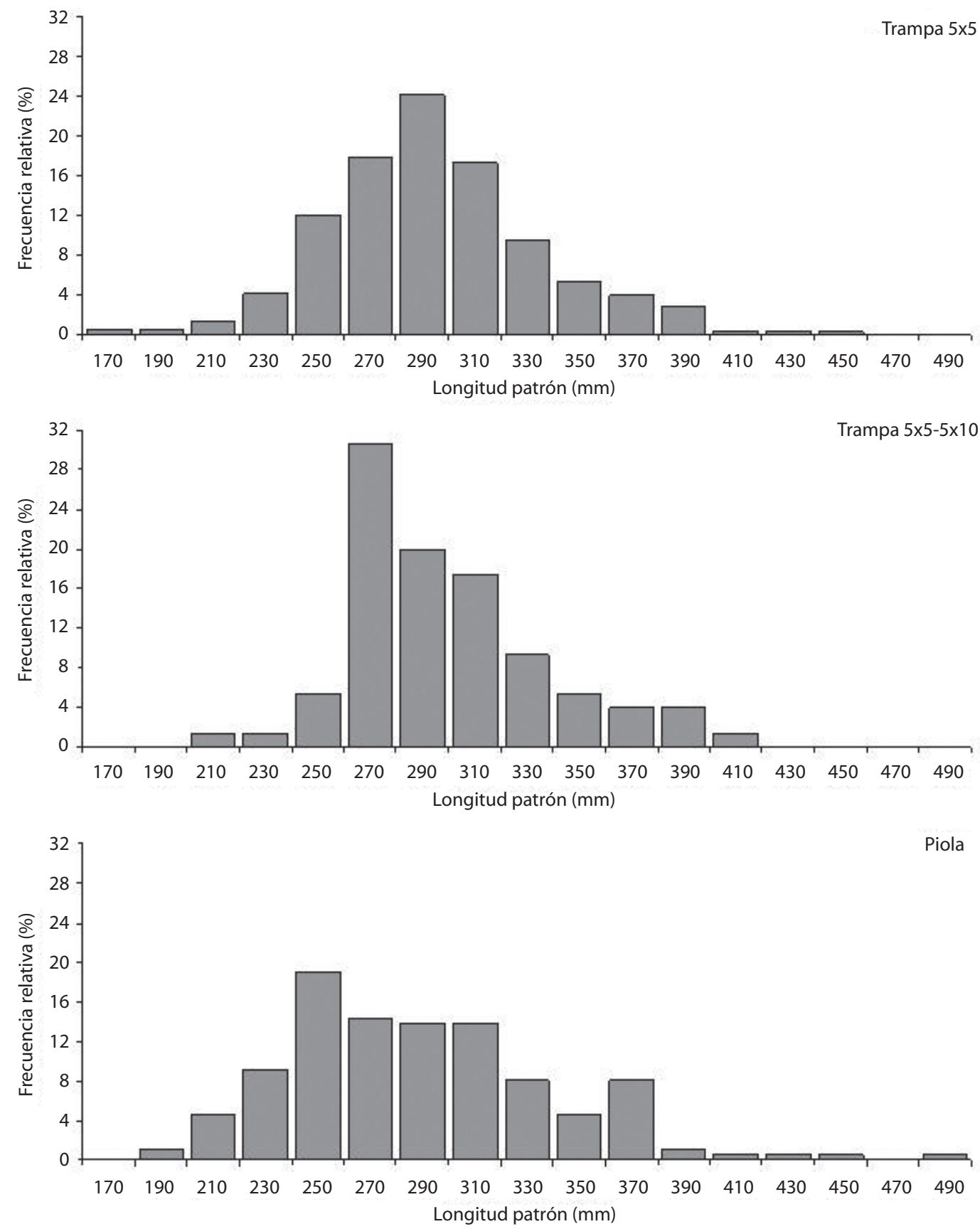

Fig. 2. Distribución de frecuencias de tallas de peces capturados en trampas y piola de mano con anzuelo en Guaymas, Sonora, México.

Fig. 2. Length frequency distribution of fish catched in traps and hook-and-line in Guaymas, Sonora, Mexico. 

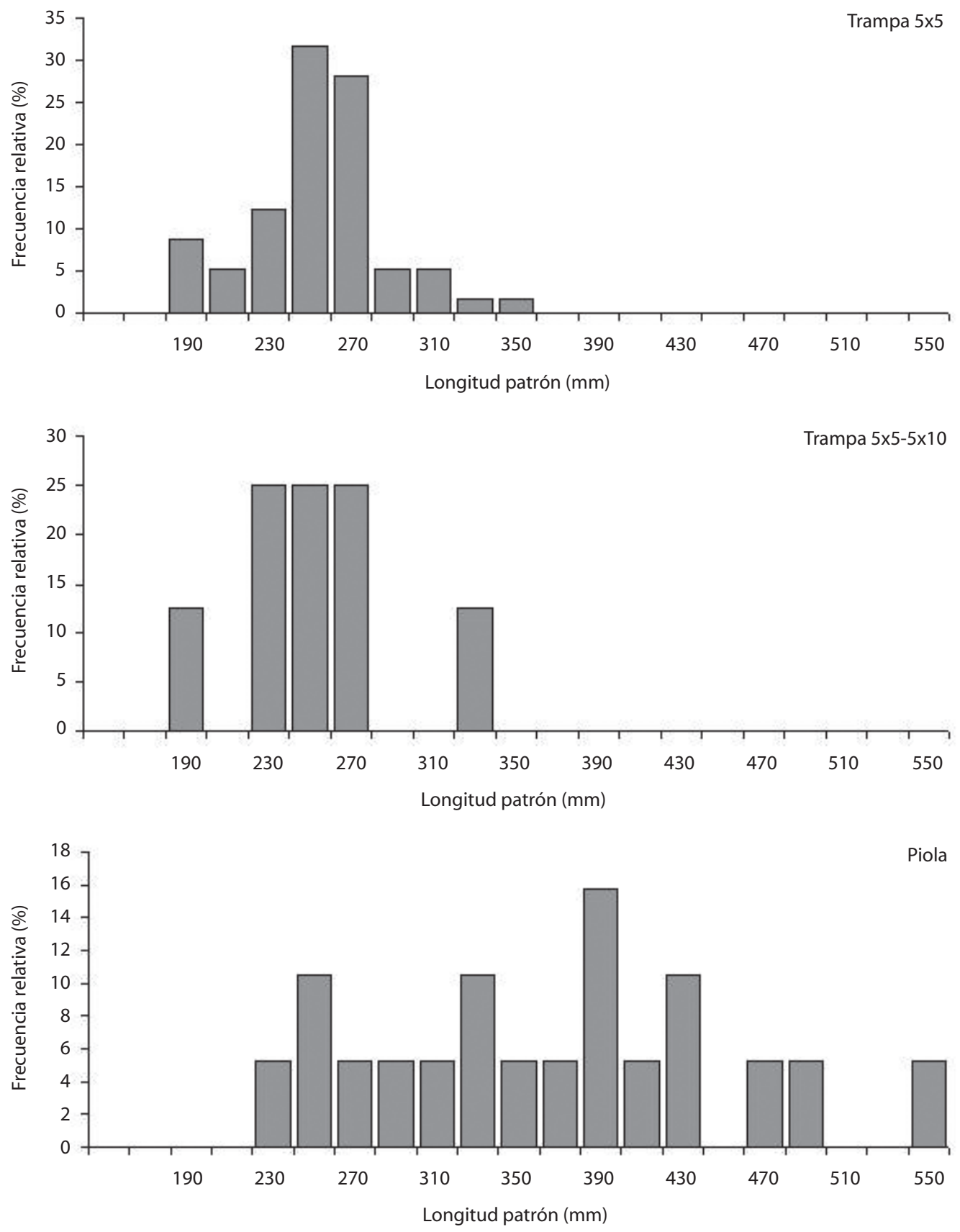

Fig. 3. Distribución de frecuencias de tallas de peces en trampas y piola de mano con anzuelo en Yavaros, Sonora, México.

Fig. 3. Length frequency distribution of fish catched in traps and hook-and-line in Yavaros, Sonora, Mexico. 

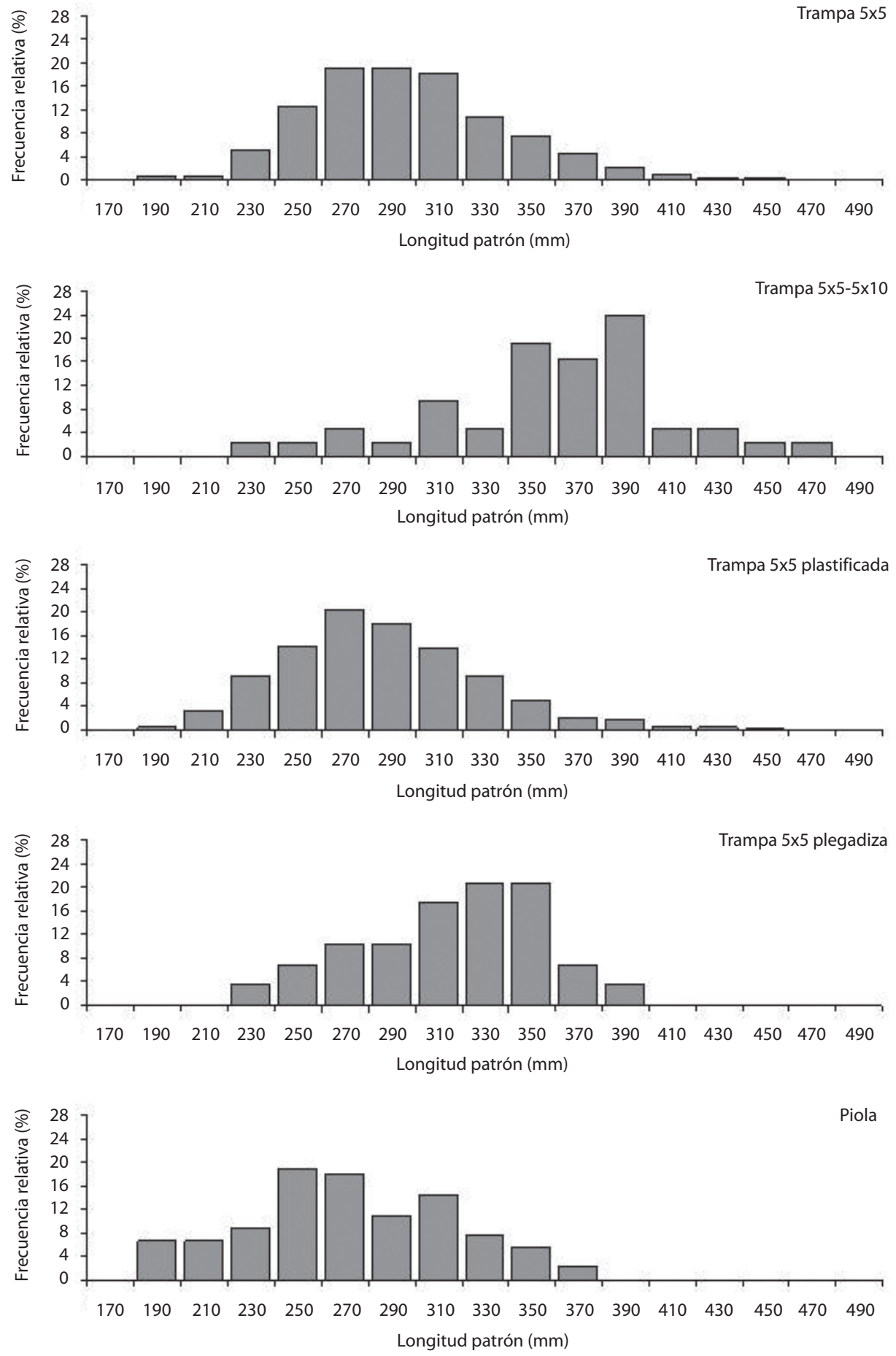

Fig. 4. Distribución de frecuencias de tallas de peces en trampas y piola de mano con anzuelo en Kino, Sonora, México.

Fig. 4. Length frequency distribution of fish catched in traps and hook-and-line in Kino, Sonora, Mexico. 

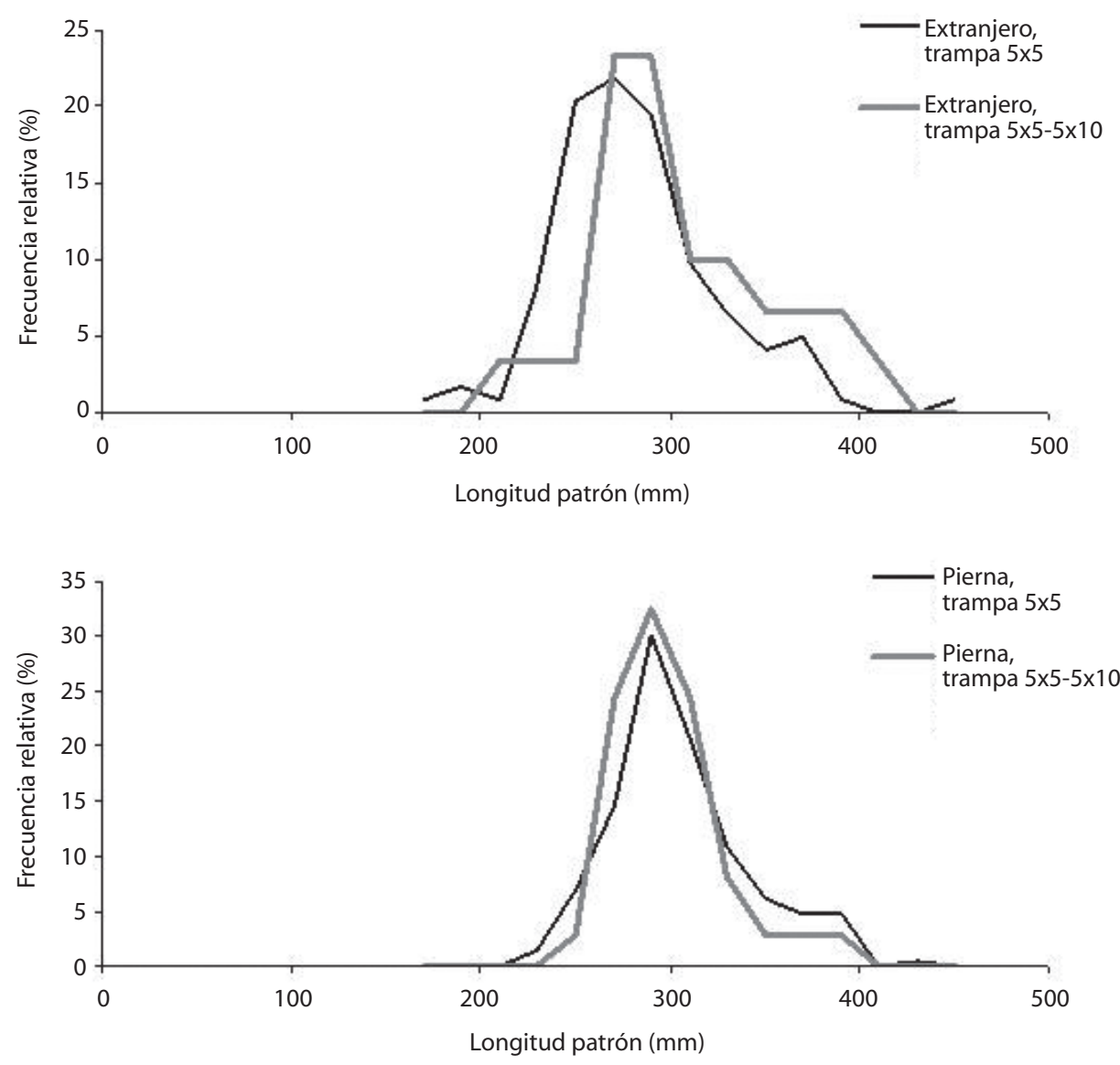

Fig. 5. Distribución de frecuencias de tallas del extranjero (Paralabrax auroguttatus) y pierna (Caulolatilus princeps) capturados en trampas en Guaymas, Sonora, México.

Fig. 5. Length frequency distribution of Paralabrax auroguttatus and Caulolatilus princeps catched in traps in Guaymas, Sonora, Mexico.

en Kino (Cuadro 2). Las curvas de selectividad obtenidas a partir de los parámetros del modelo logístico de cada tipo de trampa y para cada especie mostrada en el Cuadro 2, son presentadas en la figura 7 (Kino) y la figura 8 (Guaymas). Las curvas de selectividad de la trampa con malla más grande están corridas hacia la derecha (Fig. 7).

\section{DISCUSIÓN}

Varios trabajos han comparado las tasas de captura, la estructura de tallas y la composición de especies de las capturas en trampas de diferentes tamaños de malla pescando concurrentemente en las mismas áreas (Wolf y Chislett 1974, Olsen et al. 1978, Stevenson y Stuart-Sharkey 1980, Munro 1983, Nicholson y Hartsuijker 1983, Lockhurst y Ward 1987, Ward 1988, Bohnsack et al. 1989, Rosario y Sadovy 1991, Sheaves 1995, Robichaud et al. 1999). La tendencia es que el tamaño de malla es un factor importante en la captura de peces en trampas (Mahon y Hunte 2001), pues el número de organismos por trampa por lance decreció al aumentar el tamaño de malla, mientras que la talla media se incrementó con 

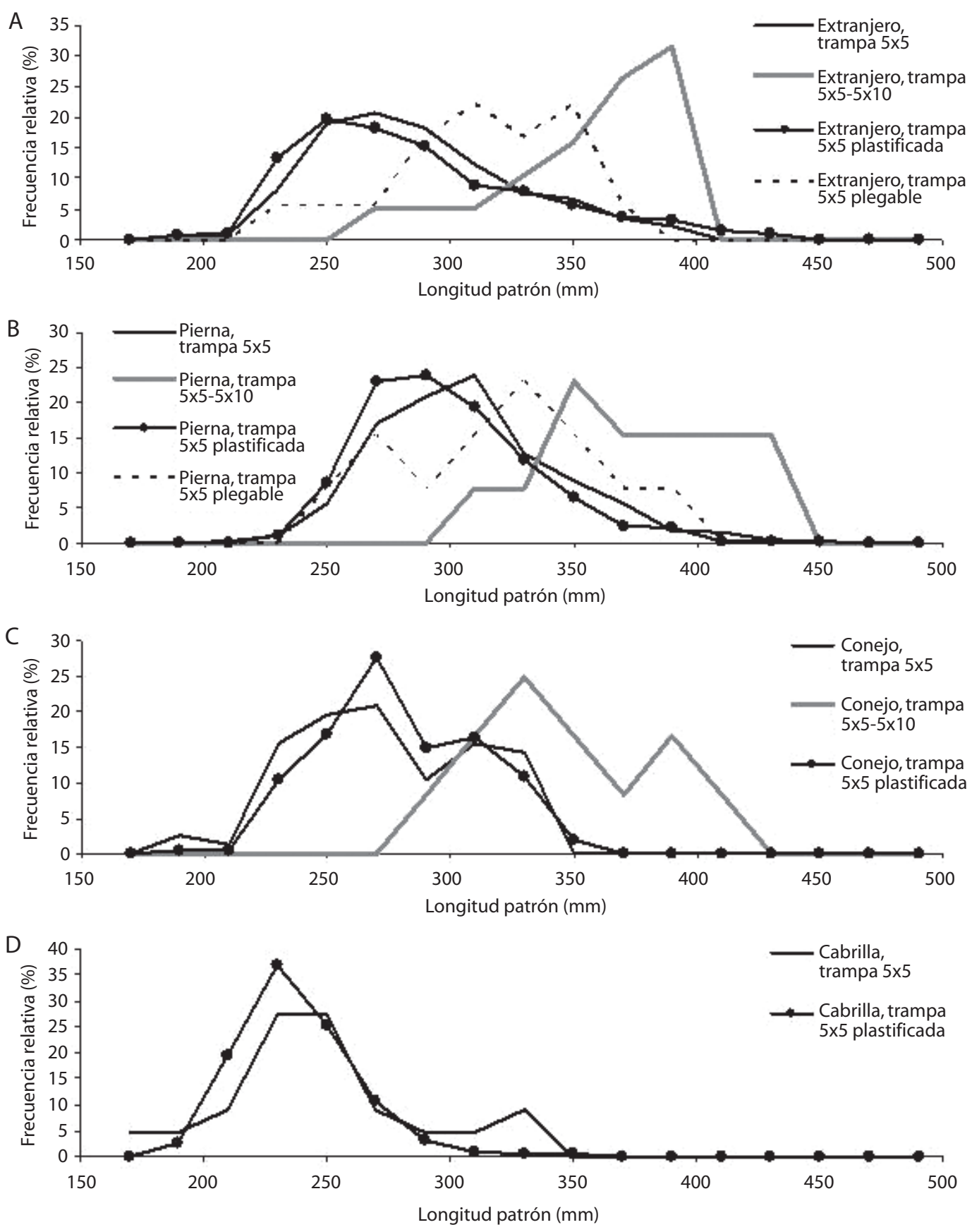

Fig. 6. Distribución de frecuencias de tallas de extranjero (Paralabrax auroguttatus), pierna (Caulolatilus princeps), conejo (C. affinis) y cabrilla (P. maculatofaciatus) capturados en trampas en Kino, Sonora, México.

Fig. 6. Length frequency distribution of Paralabrax auroguttatus, Caulolatilus princeps, P. maculatofaciatus and C. affinis catched in traps in Kino, Sonora, Mexico. 
CUADRO 2

Estimados de los parámetros de las curvas de selectividad para trampas para las principales especies, en diferentes localidades de las costas del estado de Sonora, México

TABLE 2

Selectivity curve parameter estimates for the main species in traps for different localities from the Sonora coasts, Mexico

Localidad, especie y tipo de trampa

Kino

Extranjero, trampa $5 \times 5-5 \times 10$

Extranjero, trampa $5 \times 5$ plastificada

Extranjero, trampa $5 \times 5$ plegable

Pierna, trampa $5 \times 5-5 \times 10$

Pierna, trampa $5 \times 5$ plastificada

Pierna, trampa $5 \times 5$ plegable

Conejo, trampa $5 \times 5-5 \times 10$

Conejo, trampa $5 \times 5$ plastificada

Cabrilla, trampa $5 \times 5$ plastificada

\section{Guaymas}

Extranjero, trampa $5 \times 5-5 \times 10$

Pierna, trampa $5 \times 5-5 \times 10$

-12.19574
-14.45180
-15.30180
-23.84683
-18.43312
-11.03813
-19.75065
-12.42114
-27.43913

$-13.25715$

$-13.80729$ b

0.03955
0.05955
0.05305
0.06478
0.07239
0.03566
0.06008
0.05556
0.13131

0.04519

0.04705 $\mathrm{p}$

0.49000

0.53000

0.66000

0.62012

0.69515

0.49442

0.59000

0.75546

0.93353

0.40661

0.32048
$\mathrm{L}_{50}$
$(\mathrm{~mm})$

RS

$(\mathrm{mm})$ el tamaño de malla de las trampas. Los resultados de este estudio son consistentes con estas observaciones previas. Lo anterior sugiere que el poder de pesca de las trampas puede decrecer con incrementos en el tamaño de malla. Esto se refuerza más al compararlo con la piola de mano con anzuelos, el arte de pesca principal en la región. La cantidad de peces capturados por piola por día fue menor que la obtenida en la trampa 1, pero fue mayor que la de la trampa 3 , en las tres localidades, pero fue mas notable la diferencia en Kino.

Por otro lado, en los estudios de Bohnsack et al. (1989) y Rosario y Sadovy (1991), el número de especies presentes en las capturas de trampas fue muy alto ( $\leq 60$ especies), con la mayor riqueza en las trampas de malla pequeña, tal como lo encontrado en el presente trabajo. La trampa 3 (malla grande) presentó hasta siete especies, mientras que la trampa 4 (malla pequeña) atrapó $\leq 14$ especies, muy por debajo de las 33 ó 42 especies capturadas en trampas con mallas similares en Florida y Puerto Rico (Bohnsack et al. 1989, Rosario y Sadovy 1991). Dada la composición específica observada en el presente estudio, todas las capturas de especies tuvieron valor comercial y por lo tanto pudieron ser aprovechadas. Además, ejemplares de dos de las especies sin valor comercial (anguila y tiburón gata) se pudieron liberar vivos.

Este estudio ha permitido documentar las tallas de las especies importantes capturadas en trampas para peces, aunado a las tallas obtenidas en las capturas de pesca con piola de mano con anzuelos, el cual es el arte de pesca principal utilizado para capturar a esas especies en la región. Los resultados indicaron que las tallas generales y por especie son más grandes en las trampas que las obtenidas por el método y arte de pesca actual. Esto implica que las trampas, cualquiera de ellas, tienen selectividad de tallas para peces de importancia comercial en las costas de Sonora. 

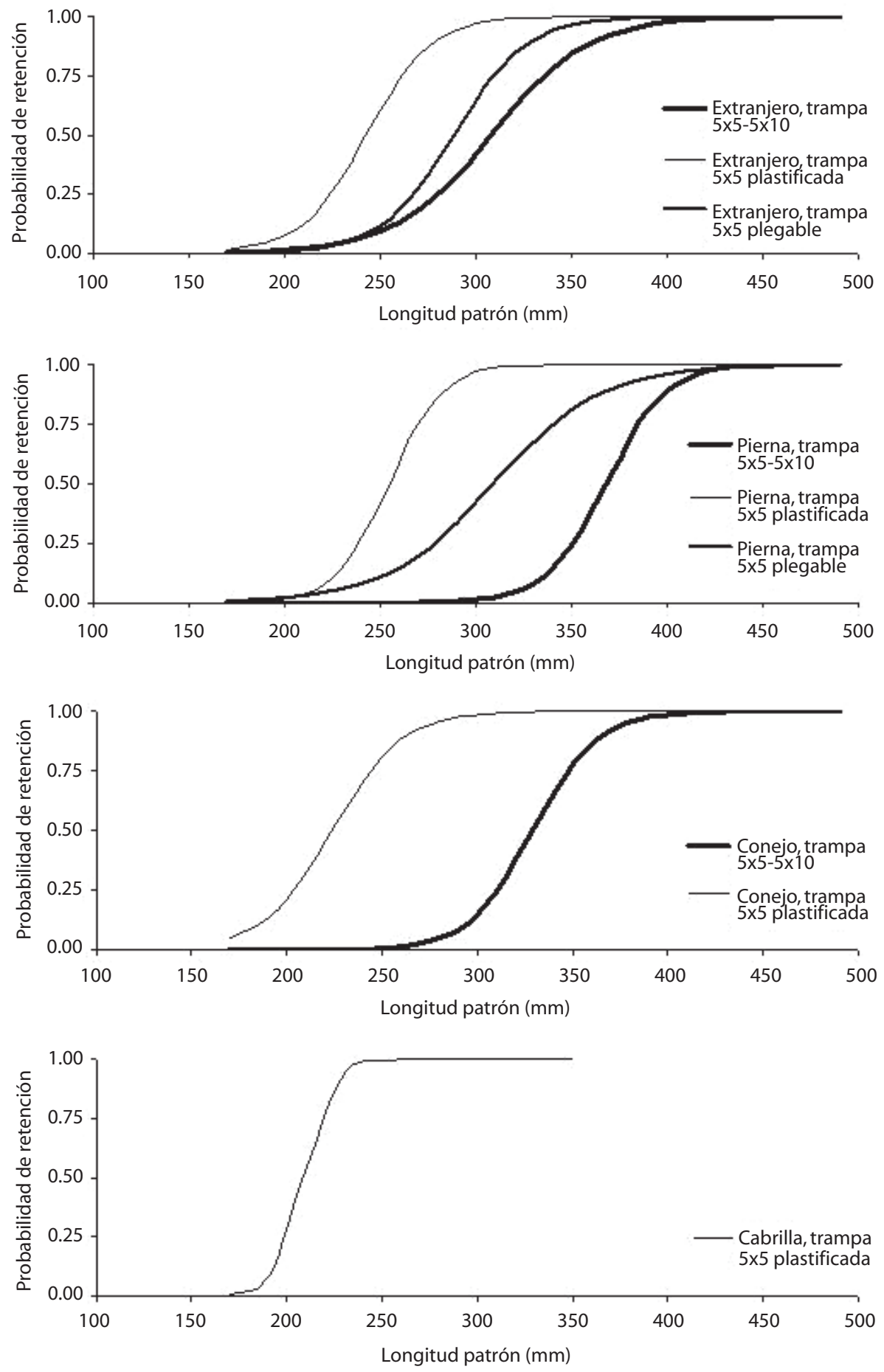

Fig. 7. Curvas de selectividad para cada tipo de trampa por especie en Kino, Sonora, México.

Fig. 7. Selectivity curves for each type of trap by species in Kino, Sonora, Mexico. 

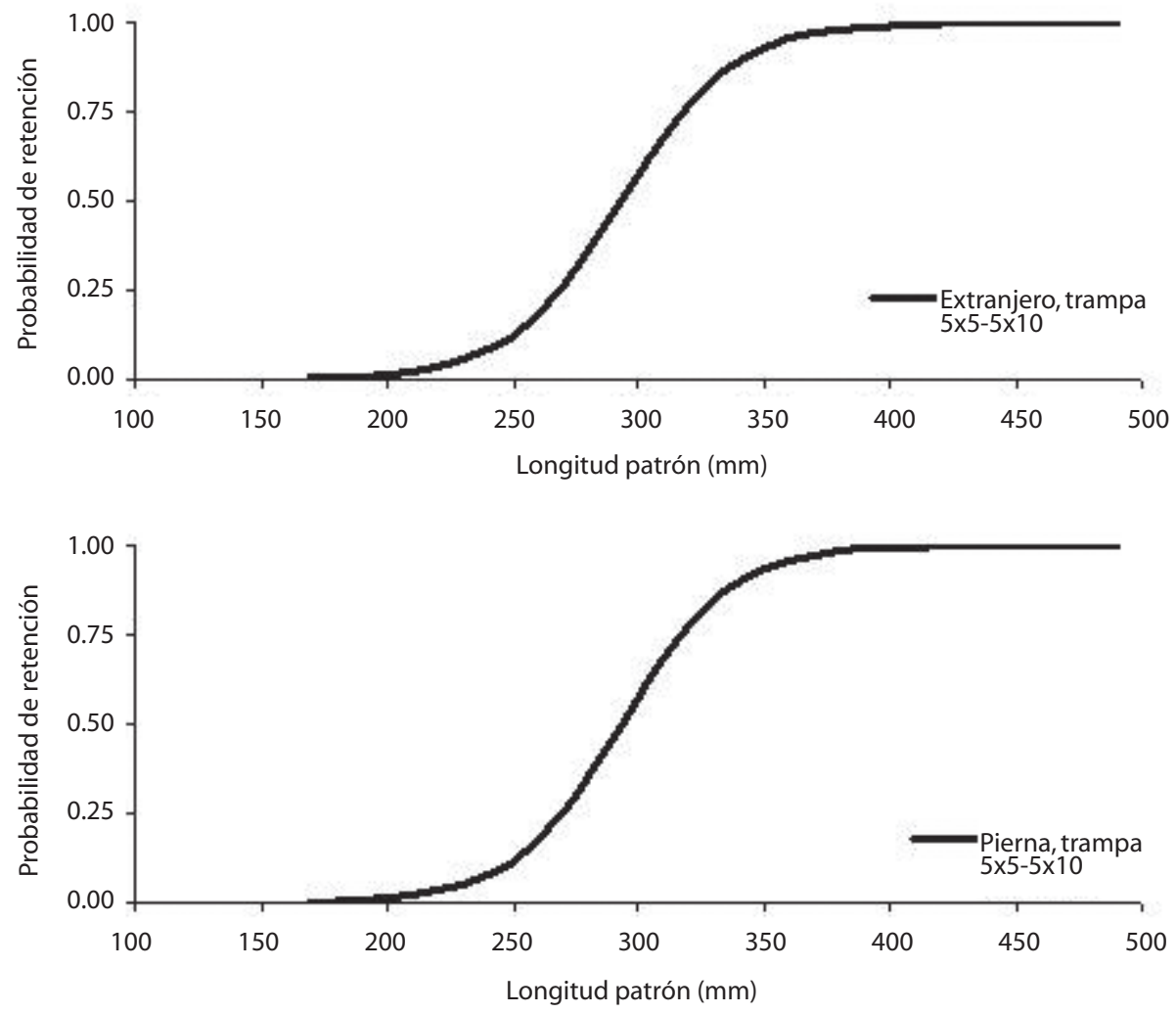

Fig. 8. Curvas de selectividad para cada tipo de trampa por especie en Guaymas, Sonora, México.

Fig. 8. Selectivity curves for each type of trap by species in Guaymas, Sonora, Mexico.

La selectividad de tallas fue obtenida a partir de experimentos de pesca comparativos sobre la suposición de que la estructura de tallas en el equipo control (trampa 1) es representativa de la población de peces en la zona de pesca (Xu y Millar 1993, Millar y Fryer 1999, Jeong et al. 2000, Stuart y Ferrell 2003, Akiyama et al. 2004). Las trampas de malla grande (trampa 3) fueron más selectivas, seguidas por la trampa 5. Esta trampa no es rígida, pues el cuerpo se construyó de paño; por lo tanto, algunas especies pueden escapar a través de esta estructura flexible (Ward 1988, Gobert 1998, Robichaud et al. 1999), aun cuando la profundidad corporal de los peces sea ligeramente mayor que la apertura máxima de la malla. La selectividad de tallas de la trampa 4 fue mas baja que el de las otras trampas, aunque considerando la estructura de tallas observada en las capturas obtenidas con la piola, es mejor que la obtenida en la piola que es el arte de pesca actualmente utilizado, es decir cualquiera de las trampas presentadas tienen una apropiada selectividad de tallas para todas las especies de peces de importancia comercial del área estudiada, sobre todo en la parte norte y centro.

Así pues, considerando los resultados (tasas de captura, estructura de tallas y especies capturadas) se puede decir que las trampas aquí estudiadas son una muy útil adición al conjunto de artes de pesca usados en la pesca ribereña de las costas de Sonora. 


\section{AGRADECIMIENTOS}

A la memoria de Francisco J. Méndez Tenorio. Agradecemos a Ángel R. Godínez Cota y Myrna L. Anguiano Carrasco por su ayuda durante el trabajo de campo y su ayuda logística. También se agradece a Enrique Morales Bojórquez por sus comentarios sobre este manuscrito. Se agradece al Fondo SAGARPACONACYT y al Instituto Nacional de Pesca (INAPESCA) por el apoyo financiero proporcionado, así como la ayuda logística dada por el INAPESCA.

\section{RESUMEN}

Se realizó un estudio orientado a evaluar tres tipos de trampas (eficiencia de captura, estructura de tallas, selectividad y composición de especies) para la captura de peces marinos en el Golfo de California. La recolecta de muestras se efectuó con trampas de $90 \mathrm{~cm}$ de ancho, $120 \mathrm{~cm}$ de largo y $50 \mathrm{~cm}$ de alto, con malla galvanizada cuadrangular en tres tamaños: una trampa completamente construida con abertura de malla de 5 x $5 \mathrm{~cm}$ (trampa 1); otra con malla de $5 \times 5 \mathrm{~cm}$, pero con el panel trasero de $5 \times 10 \mathrm{~cm}$ (trampa 2) y la tercera con malla de $5 \times 10 \mathrm{~cm}$ (trampa 3 ). Los experimentos se llevaron a cabo entre octubre del 2005 y agosto del 2006. Las especies que presentaron mayor incidencia de captura en cuanto a proporción del volumen total fueron el extranjero (Paralabrax auroguttatus), pierna (Caulolatilus princeps), cabrilla arenera (P. maculatofaciatus) y conejo (C. affinis) y prácticamente todas las capturas tuvieron valor comercial. El número de organismos por trampa por lance decreció al aumentar el tamaño de malla, mientras que la talla media se incrementó con el tamaño de malla de las trampas. Además las tallas son más grandes en cualquiera de las trampas que las obtenidas con piola de mano con anzuelos (el arte de pesca autorizado). Los resultados de la selectividad de tallas indicaron que las trampas de malla grande fueron más selectivas que las de malla más pequeña. En consecuencia, la captura con trampas es mejor que con los anzuelos y línea de mano, por lo que las trampas serían una muy útil adición al conjunto de artes de pesca usados en la pesca artesanal de la costa de Sonora, México.

Palabras clave: trampas para peces, anzuelos, selectividad de trampas, golfo de California, México.

\section{REFERENCIAS}

Akiyama, S., S. Kaihara, T. Arimoto \& T. Tokai. 2004. Size selectivity of a trammel net for oval squid Sepioteuthis lessoniana. Fish. Sci. 70: 945-951.
Anónimo. 2000. Carta Nacional Pesquera (Segunda Sección). Diario Oficial de la Federación. Secretaría de Gobernación, México, D. F., 28 de agosto del 2000.

Bullock, L. H. \& M. D. Murphy. 1994. Aspects of the life history of the yellowmouth grouper, Mycteroperca interstitialis, in the eastern Gulf of México. Bull. Mar. Sci. 55: 30-45.

Bohnsack, J. A., D. L. Sutherland, D. E. Harper, D. B. McClellan, M. W. Hulsbeck \& C. M. Holt. 1989. The effects of fish trap mesh size on reef fish catch off south eastern Florida. Mar. Fish. Rev. 51: 36-46.

Fischer, W., F. Krupp, W. Schneider, C. Sommer, K. E. Carpenter \& V. H. Niem (eds.). 1995. Guía FAO para la identificación de especies para los fines de la pesca. Pacifico centro-oriental. Vols. I, II y III. FAO, Roma, Italia.

Gobert, B. 1998. Density-dependent size selectivity in Antillean fish traps. Fish. Res. 38: 159-167.

Helser, T. E., J. P. Geaghan \& R. E. Condrey. 1998. Estimating gillnet selectivity using nonlinear response surface regression. Can. J. Fish. Aquat. Sci. 55: 13281337.

Hovgard, H. 1996. A two-step approach to estimating selectivity and fishing power of research gillnets used in Greenland waters. Can J. Fish. Aquat. Sci. 53: 1007-1013.

INP. 1976. Catalogo de Peces Marinos Mexicanos. Secretaria de Industria y Comercio. Subsecretaria de Pesca, Instituto Nacional de la Pesca. México, D. F., México.

Jeong, E. C., C. D. Park, S. W. Park, J. H. Lee \& T. Tokai. 2000. Size selectivity of trap for male red queen crab Chionoecetes japonicus with the extended SELECT model. Fish. Sci. 66: 494-501.

Luckhurst, B. \& J. Ward. 1987. Behavioral dynamics of coral reef fishes in Antillean fish traps at Bermuda. Proc. Gulf Carib. Fish. Inst. 38:528-546.

Mahon, R. \& W. Hunte. 2001. Trap mesh selectivity and the management of reef fishes. Fish Fish. 2: 356-375.

Manickchand-Heileman, S. C. \& D. A. Phillip. 2000. Age and growth of the yellowedge grouper, Epinephelus flavolimbatus, and the yellowmouth grouper, Mycteroperca interstitialis, off Trinidad and Tobago. Fish. Bull. 98: 290-298.

Millar, R. B. 1992. Estimating the size-selectivity of fishing gear by conditioning on the total match. J. Am. Stat. Assoc. 87: 962-968. 
Millar, R. B. 1995. The functional form of hook and gillnet selection curves cannot be determined from comparative catch data alone. Can. J. Fish. Aquat. Sci. 52: 883-891.

Millar, R. B. \& S. J. Walsh. 1992 . Analysis of trawl selectivity studies with an application to trouser trawls. Fish. Res. 13: 205-220.

Millar, R. B. \& R. J. Fryer. 1999. Estimating the sizeselection curves to towed gears, traps, nets and hooks. Rev. Fish Biol. Fish. 9: 89-116.

Miller, D. J. \& R. N. Lea. 1972. Guide to the coastal marine fishes of California. Calif. Dept. Fish and Game, Fish. Bull. California, EEUU.

Munro, J. L. (ed.). 1983. Caribbean coral reef fishery resources. ICLARM Studies and Reviews 7 . ICLARM, Manila, Filipinas.

Nevárez-Martínez, M. O., A. Balmori-Ramírez, E. MirandaMier, F. J. Méndez-Tenorio, C. Cervantes-Valle, M. L. Anguiano-Carrasco \& J. P. Santos-Molina. 2005. Diseño y construcción de trampas para peces marinos en el Golfo de California. Informe Técnico, Instituto Nacional de la Pesca, CRIP Guaymas. Guaymas, Sonora, México..

Nicholson, W. \& L. Hartsuijker. 1983. The state of the fisheries resources of the Pedro Bank and South Jamaica Shelf, p. 215-254. In National reports and selected papers presented at the third Session of the Working Party on Assessment of Marine Fishery Resources (Western Central Atlantic). FAO Fish. Rep. 278 (Suppl.). FAO, Roma, Italia.

Olsen, D. A., A. E. Dammann \& J. A. LaPlace. 1978. Mesh selectivity of West Indian fish traps. Mar. Fish. Rev. 40:15-16

Pope, J. A., A. R. Margetts \& J. M. Hamley. 1975. Manual of methods for fish stock assessment Part III. Selectivity of fishing gear. FAO Fish. Tech. Paper 41. FAO, Roma, Italia.

Robichaud, D., W. Hunte \& H.A. Oxenford. 1999. Effects of increased mesh size on match and fishing power of coral reef fish traps. Fish. Res. 39: 275-294.
Rosario, A. \& Y. Sadovy. 1991. Trap Mesh Selectivity off the West Coast of Puerto Rico (January, 1990-December, 1990). Fisheries Research Laboratory, Department of Natural Resources. Mayagüez, Puerto Rico.

Sheaves, M. J. 1995. Effect of design modifications and soak time variations on Antillean-Z fish traps performance in a tropical estuary. Bull. Mar. Sci. 56: 475-489.

Smith, C. L. 1971. A revision of the American grouper: Epinephelus and allied genera. Bull. Am. Mus. Nat. Hist. 146: 69-241.

Stevenson, D. K. \& P. Stuart-Sharkey. 1980. Performance of wire fish traps on the western coast of Puerto Rico. Proc. Gulf Caribb. Fish. Inst. 32:173-193.

Stewart, J. \& D. J. Ferrell. 2003. Mesh selectivity in the New South Wales demersal trap fishery. Fish. Res. 59: 379-392.

Tokai, T. 1997. Maximum likelihood parameter estimates of a mesh selectivity logistic model through SOLVER on MS-Excel. Bull. Jap. Soc. Fish. Oceanogr. 61: 288-298.

Thomson, D. A., L. T. Findley \& A. N. Kerstitch. 1979. Reef Fishes of the Sea of Cortez. Wiley, Nueva York, EEUU.

Ward, J. 1988. Mesh size selection in Antillean arrowhead fish traps, p. 455-467. In S. Venema, J. MollerChristensen \& D. Pauly (eds.). Contributions to Tropical Fisheries Biology. FAO Fish. Rep. 389. FAO, Roma, Italia.

Wileman, D. A., R. S. T. Ferro, R. Fonteyne \& R. B. Millar. 1996. Manual of methods of measuring the selectivity of towed fishing gears. ICES Coop. Res. Rep. 235: $126 \mathrm{p}$.

Wolf, R. S. \& G. R. Chislett. 1974. Trap fishing explorations for snapper and related species in the Caribbean and adjacent waters. Mar. Fish. Rev. 36: 49-61.

Xu, X. \& R. B. Millar. 1993. Estimation of trap selectivity for male snow crab (Chionoecetes opilio) using the SELECT modeling approach with unequal sampling efforts. Can. J. Fish. Aquat. Sci. 50: 2485-2490. 
